\title{
RaIlway BREAK.
}

After the Meeting a model of a Railway Break was exhibited by Mr. W. G. Creamer, of New York. This plan was stated to embrace, first, an arrangement of compensating, or balanced breaks, worked by one lever, and distributing the pressure equally on all the wheels, with a small expenditure of power. Secondly, an attachment of reserved power to the breaks of every carriage, in combination with a non-elastic signal rope, so as to enable the driver instantly to apply every break in case of danger; or on the separation of the train, or any part being thrown from the line, the breaks would apply themselves automatically, as each carriage was entirely independent of all the others, and the breaks were available wherever they were placed in the train. 